\author{
Proceedings of the $10^{\text {th }}$ International Conference on Applied Informatics \\ Eger, Hungary, January 30-February 1, 2017. pp. 235-243 \\ doi: $10.14794 /$ ICAI.10.2017.235
}

\title{
Modeling and printing in 3D at higher education
}

\author{
Ildikó Papp, Marianna Zichar \\ University of Debrecen, Faculty of Informatics, Debrecen, Hungary \\ papp.ildiko@inf .undideb.hu, zichar.marianna@inf.unideb.hu
}

\begin{abstract}
The technology for 3D printing, also known as additive manufacturing, is older than many people think. This decade brought a real breakthrough in this field which can be certified by the dynamically increasing number of $3 \mathrm{D}$ printers. The vision of certain researchers and manufacturers is that $3 \mathrm{D}$ printing will spread as widely as scanning did in the last century. Nevertheless, 3D printing requires much knowledge, so without the support of education its spread is impossible. This paper aims to give an overview of what and how to teach about 3D modeling and 3D printing in higher education. It also reveals our experience gained during one-semester-long courses held at the University of Debrecen Faculty of Informatics.
\end{abstract}

Keywords: higher education, 3D printing, 3D modeling

MSC: $68 \mathrm{U} 07$

\section{Introduction}

Objects around us make up our lives for thousands of years. In the 21st century, most of these objects were purchased in an appropriate store or sometimes were created manually by professionals or amateurs or sometimes by ourselves. Manual manufacturing means that we can have a chance that our own needs will be taken into consideration. Let us think on traditional tailors whose task is to sew garments fitting the customer properly. Nowadays, customization appears in many forms (Tshirts, mugs, keyrings, and other memories with photo provided by the customer) as a service, which can be even online. No doubts, that society requires this kind of personalization which is widened by the appearance of $3 \mathrm{D}$ printing. 3D printing technology changes the dynamics of consumer culture by turning users into active creators from passive consumers. But without any knowledge about 3DP this revolution cannot come true, so it is high time to introduce the basics of 3DP into the education. This paper aims to give an overview about the experience of authors 
gained during the first year of having courses about 3D printing at the Faculty of Informatics University of Debrecen.

\section{3D printing}

Technology of 3D printing (3DP) belongs to the family of additive manufacturing and it is often identified with it. Basically, 3DP is a process where a physical object is made based upon a three-dimensional digital model, typically by superimposing several thin layers of a material. Unlike subtractive manufacturing, we tend to impose material to only location, where the material builds up the object to be printed. The applied 3DP technology determines whether this expectation can be met or not, but comparing 3DP to subtractive manufacturing there is a significant difference between the amount of waste material.

It was the rapid prototyping that used first additive manufacturing during the late 1980s and 1990s. Nowadays its application does not limited into producing prototypes, but 3D printers are also used to create finished high-quality products. Each object is built up uniquely, that is why $3 \mathrm{D}$ printing is especially great for making unique and customized items, or small series of objects.

Beyond the traditional application (consume electronics, surgical guides and prototypes, medical implants, component parts), 3DP is also used for jewelry and fashion design and can support the education in many ways. Fortunately, the price of desktop 3D printers has decreased largely, so it is not an everlasting dream of educational institutions to have one. The most popular and cost-effective printers use PLA as a printing material and based on Fused Deposition Modeling that will be overviewed in the next section.

\subsection{Fused Deposition Modeling}

$3 \mathrm{D}$ printing technologies can be categorized into four groups based on the main property or printing material: filament-based, resin-based, powder-based and multicolor 3D printing. Fused Deposition Modeling (FDM) is a filament (thermoplastic wound into rolls) based 3DP technology popularly featured in most consumer grade desktop 3D printers. This is the typical printer that educational institutions can have, so other resources are recommended to get to know their operation principles [1].

FDM technology is about feeding the plastic filament through a nozzle which heats up it to a semi-liquid state. This is then deposited along a defined extrusion path on top of the build platform or the previous layer. Repeating of deposition creates up the $3 \mathrm{D}$ object by moving down the build platform. The most popular filament is called Polylactic Acid (PLA) that is biodegradable and made from dextrose by fermentation [2]. This is the reason for calling it often "the green plastic". We have to mention Acrylonitrile Butadiene Styrene (ABS) as well, although its usage requires a heated build platform, which is not offered by all of the printers based on FDM [1]. 


\subsection{D printing process}

Although it seems rather attractive to take our idea into physical shape, its process is not so trivial and contains many pitfalls. This section provides a general overview about the most generic steps of 3D printing to emphasize its complexity.

\subsubsection{How to get a $3 \mathrm{D}$ model?}

There are several possibilities to have a $3 \mathrm{D}$ model. The most simple one is download from an online repository which can be even free. Currently, Thingiverse is ranked as the most popular site offering thousands of $3 \mathrm{D}$ models [3]. If you need a replica of an object, 3D scanners can help you to create the digital model of your object. Follow-up editing may be required, of course. The most time-consuming and difficult task is to design the model from the scratch by yourself using a 3D modeling software. Not only commercial, but free ones are also available ensuring the opportunity of design for hobbyists of any age. The decision about which software to be used is often influenced by existing working experience gained by any kind of project [4]. Just to mention some popular free design products:

- TinkerCAD

It is an amazing tool that enables one to make 3D designs and render 3D models in a web browser. The application uses Constructive Solid Geometry (CSG) that allows one to create a complex solid by using Boolean operators to combine simple objects. Not only can one make objects, but he or she can also save, share and transform models into STL files for 3D printing. Also, TinkerCAD is a community site where users can consult and support each other. Many tutorials are available for novice designers [5].

- SketchUp

It is considered to be one of the simplest modeling software to use although experts say that it facilitates design of complicated models as well. It is often recommended for hobbyist, but seasoned 3D designers, experienced users, or artists prefer it too. It has an easy learning curve that allows even beginners to create models effortlessly and than it offers the user the ability to draw highly complex models. SketchUp works best on rectilinear and geometrictype models, but can create curvy, organic models of lower quality [6].

\section{- Blender}

It is a free and open source $3 \mathrm{D}$ creation suite. It supports the entirety of the 3D pipeline-modeling, rigging, animation, simulation, rendering, compositing and motion tracking, even video editing and game creation. Blender gives its users enormous design freedom with nearly innumerable tools. It is known for its steep learning curve and is often considered difficult for beginners. Nevertheless, today's most popular free modeling software related to $3 \mathrm{D}$ printing [7]. 


\section{- OpenSCAD}

It is a software for creating solid 3D objects based on program codes instead of visual interaction. Design of the models is described within a text file, that it parses to generate and show 3D shapes. Concerning the modeling itself both principles of CSG and extrusion of 2D primitives can be applied. OpenSCAD provides not only parameterized commands to create 3D solids, but also models with surfaces determined by functions like $\mathrm{z}=\mathrm{f}(\mathrm{x}, \mathrm{y})$ can be constructed as well [8].

\subsubsection{Why to convert the model into an STL file?}

Your CAD model gained by any way mentioned in the previous subsection describes the geometry of the object as a solid. In contrary, an STL file is a triangulated representation of a $3 \mathrm{D} \mathrm{CAD}$ model. The triangulation of a surface will cause faceting of the 3D model. An STL file is a simple series of $\mathrm{x}, \mathrm{y}$ and $\mathrm{z}$ coordinate triplets describing triangular facets supplemented by the surface normal of each facet. STL is the standard file type used by most 3D printers.

\subsubsection{How to create layer-by-layer information?}

There is still one step before starting the printing process because of the operating principle of 3DP. What we need is information about the path of extruder where it has to deposit the material. So called slicer programs are used for this purpose which is provided by the producer of the $3 \mathrm{D}$ printer. We have to mention that free slicers are also available so we can select the one that suits our needs the best. We only have to check the output file format of the slicer to ensure that the printer accept it or not. Settings made in the slicer influence the final object greatly, that is why next subsection provides an overview about the most common settings.

\subsection{Settings influencing the printing}

Before starting the slicing a number of settings have to be done in order that the quality of the printed object meets our requirements. Raft serves as a basement of the model and helps fixing it in the specified position. Applying support makes it possible to deal with slopes, bridges and overhangings. Both of them have to be removed after printing and obviously can result some waste. Number of shells determines the number of drawing the outline of the model. Infill rate (varying from $0 \%$ to $100 \%$ ), layer thickness affect the mechanical strength. Orientation is rather important and can influence further settings (for example need of support sometimes can be eliminated by changing the orientation of the model).

\section{3DP in education}

Nowadays, we are experiencing a period of educational reforms, which includes the spread of experience-centered teaching methods as well. STEAM is a special educa- 
tional approach to learning that applies Science, Technology, Engineering, the Arts and Mathematics as access points for guiding student inquiry, dialogue, and critical thinking. The students work through the creative process, which strengthen different skills like this engaging in experiential learning, persisting in problem-solving or embracing collaboration. 3D printing is an excellent tool for teaching based on STEAM idea. Thereby educational institutions should adopt 3DP technology at every level from elementary school to university. 3D printing companies have seen the potential of education and thus support educational institutions by different ways.

Stratasys Ltd. (the owner of MakerBot brand) has provided FDM 3D printers to more than 5,000 schools throughout the United States, and others around the world. MakerBot's motto is: "Empowering Today's Students to Become the Innovators of Tomorrow." They launched the program titled MakerBot in Education and published a handbook MakerBot in the Classroom [9, 10]. MakerBot offers a full set of solutions and different services that enable students to tackle real-world problems - at any grade level. Educators can connect to communities on websites Thingiverse and Thingiverse Education, which are rich in content and access tools that are easy to deploy [3, 11]. Recently, many additional corporations (General Electric, Samsung, Sony, etc.) have already joined this initiation, support this propagation, and launched their own educational programs.

\section{3DP at the Faculty of Informatics UD}

In the last year, FreeDee Ltd., as the authorized distributor of MakerBot products in Hungary, made a call titled MakerBot at the Classroom. Educational institutions at any level could apply for having a desktop 3D printer. First, 11 printers were distributed among the winners; a bit later another two Hungarian companies, CraftUnique Ltd. and Leopoly Ltd., joined the innovative initiative 3DTECH at Schools and offered additional printers. As one of the winners, Faculty of Informatics at the University of Debrecen has become the owner of a 3D printer of kind MakerBot Replicator 5th Generation. Starting from this date, our ideas and efforts have been published in our own blog in Hungarian [12]. This section briefly presents how 3D printing and modeling have appeared in regular education and in promotion of science.

\subsection{Optional course in the education}

After getting the initial experience, our team has taken significant steps to ensure that 3D printing and additive manufacturing become part of under-, and postgraduated education at the Faculty of Informatics. The optional course Introduction to $3 D$ Printing and Modeling is available for students of Business Informatics, Computer Science and Computer Science Engineering undergraduate programs. The course is structured as a 14-week-long course with weekly meetings. Each meeting 
is held in a laboratory and takes two hours.

The course covers the following topics:

- Historical overview of 3DP, characteristics of different technologies and materials.

- The principles of 3D printing and the basics of additive manufacturing.

- Getting to know the preparation for printing: slicing and overview of 3DP's printing options.

- Using repositories and databases to get 3D models, post-processing.

- 3D modeling principles: sketches, sketch-based operations to create 3D objects, primitives, operations with primitives, CSG model.

- Advanced practices for more detailed models and assemblies: transformations, scaling object-based operations to finish models (e.g. chamfer, fillet, linear and circular pattern operators, mates).

- Requirements for printable models: dimensions, watertight models, movable parts, gaps, wall width, repairing STL mistakes, mesh technologies.

During the semester, students have to prepare several models designed by themselves which are to be printed as well. Of course, we provide an opportunity to consult on ongoing and we offer assistance in printing too [12].

After completing the course, the students will be able to solve different modeling tasks and prepare the downloaded or self-designed models for 3D printing. The spatial orientation ability and problem-solving skills of theirs are improved spectacularly. The possibility of 3D printing awakes the youngsters' creative desire, encourages experimentation and also creates the possibility of acquiring the theoretical background.

The course is available for students of IT teacher degree program as well. We considered this to be important as they will be future teachers and educate next generations. 3D printing has some nice features which can revolutionize education. It can brings out objects from the digital screen of the computer into the hands of the students for inspection or analysis. In that way, a connection can be set up between the physical and the digital world. Therefore the teachers can make the education more fun, participatory and lively [13, 14].

\subsection{Other appearance forms of STEAM practices}

Faculty of Informatics is actively involved in promoting sciences and technologies. Several events are organized by our university but we are happy to join national or European initiatives as well. Our lectures, presentations, workshops are very popular among the audience of various ages. Events from the last year: 
- Researchers' Night

It is the most popular science promotional event is Europe. Our Faculty joined this initiative 5 years ago. Our 3D printer kept working that afternoon and several modeling classes could be attended this afternoon.

- Girls in ICT day

This initiative is a global effort to encourage girls and young women to consider studies and careers in information and communication technologies (ICT). Our faculty is ready to host the girls before their career choice and help them to make their decision by organizing some rather exciting workshops.

- Science on Stage Hungary

Science on Stage Europe provides a European platform for science teachers to exchange teaching concepts and to share ideas. The ultimate goal is to improve science teaching by encouraging creativity in science teachers. Thereby, the movement can encourage more schoolchildren to consider a career in science or engineering by spreading good teaching concepts among Europe's science teachers. In the last year we participated as an exhibitor and workshop holder at this event that was also the preselection for the European festival.

- NI Academy - For the experts of the future

This is a series of lectures aimed at promoting informatics and engineering activities and supported by National Instruments Hungary Ltd. and Science Center Agóra. During the event, we held a lecture for 300 students about the possibilities of $3 \mathrm{D}$ printing.

- Digital Theme Week

The general aim of theme weeks is to change attitudes towards public education of 21st century, to play and experience the practical skills that can be used in everyday life, and to encourage teachers to try new pedagogical methods. The Digital Theme Week was organized for the second time in Hungary. We had 35 hours of lectures, presentations and modeling exercises for students of different ages from 10 to up.

- TechGirls

The TechGirls series was held for the first time with the aim of giving high school girls the opportunity to meet universities, colleges and companies presenting exciting technical faculties with various technical and scientific tools. The Faculty of Informatics had an independent stand, where 3D printing received special attention.

- Campus festival

The Campus Festival is the largest summer party in Eastern Hungary, which is especially supported by the University of Debrecen. In addition to the best-known domestic and foreign star performers, humorists and actors, the university awaits visitors with a separate event venue. The University Square 
provides an opportunity to introduce faculties and their educational and research activities, and they call young people and families a playful pastime. The 3D technologies - because of their spectacular - always appear in our stand.

\section{- School trips to Faculty of Informatics}

Secondary schools put an increasing pressure on the Faculty of Informatics to provide a professional program as part of a school trip. Presenting the $3 \mathrm{D}$ printing and supercomputing are the most popular program items in this offer.

\section{Conclusions}

It is obvious that new technologies bring revolution to our life and require new knowledge from the would-be employees. It is a good strategy if the educational system of a country can react to these kinds of challenges and tries to involve the basics of the new technologies at least into the higher education. Another important role of the stakeholders to motivate the youngsters to get to know as many things as they can in order to be able to find their real profession which will provides them the feeling of satisfaction. 3D printing and modeling is exactly a special field, which can be of high interest among the different generations. Even if we have no $3 \mathrm{D}$ printer, it is worth to introduce the technology to the students which gives them extra motivation to deal with STEAM subjects. Different events also have to be organized to bring the technology and knowledge behind it closer to youngster. Our experience gained since the faculty won the printer justifies our thoughts about how important to involve new technologies into education.

\section{References}

[1] Horvath, J., Mastering 3D Printing: Modeling, printing and prototyping with reprap-style 3D printers, Apress, (2014)

[2] Giges, S., Nancy, Top 10 Materials for 3D Printing, ASME.org, http://amse.org/, (2014), Last accessed: 21.05.2017.

[3] Thingiverse, MakerBot Industries, https://www.thingiverse.com/, Last accessed: 21.05.2017.

[4] Novac, M., Nova, O., Indrie, L., Using CAD programs for computation of the 3D transient temperature distribution, Proceeding of 5th International Conference on Electromechanical and Power Systems, Republic of Moldova, (2005), 904-906, ISBN: 9737162307.

[5] Kelly, J.F., 3D Modeling and Printing with Tinkercad: Create and Print Your Own 3D Models, Pearson Education, (2014).

[6] Ritland, M. 3D Printing with SketchUp, PACKT Publishing, (2014).ISBN 9781783284573 
[7] BAcker, F., Top 25: Most Popular 3D Modeling \& Design Software for 3D Printing, https://i.materialise.com/blog/ top-25-most-popular-3d-modeling-design-software-for-3d-printing/, (2015), Last accessed: 21.05.2017.

[8] Francois, J., How to use OpenSCAD (1), tricks and tips to design a parametric 3D object, http://www.tridimake.com/2014/09/ how-to-use-openscad-tricks-and-tips-to.htm, (2015), Last accessed: 21.05.2017.

[9] Amundsen, M., Arden, E., Lentz, D., Lyttle, P., Tahlman, L., MakerBot in The Classroom, An Introduction to 3D Printing and Design, MakerBot Publishing, Brooklyn, NY, (2015), ISBN: 9781495161759

[10] MakerBot in Education, MakerBot Industries, https://www.makerbot.com/ education/, Last accessed: 21.05.2017.

[11] Thingiverse Education, MakerBot Industries, https://www.thingiverse.com/ education/, Last accessed: 21.05.2017.

[12] PAPP, I., 3D-ben printelődIK, https://3dbenprintelodik.blogspot.hu/, Last accessed: 21.05.2017.

[13] Importance of 3D Printing in Education, Educational Technology and Mobile Learning, http://www.educatorstechnology.com/, Last accessed: 21.05.2017.

[14] Peels Joris, 3D Printing in Education: How Can 3D Printing Help Students?, 3DPRINT, http://www.3dprint.com, Last accessed: 21.05.2017. 